\title{
Unsupervised feature extraction applied to bioinformatics
}

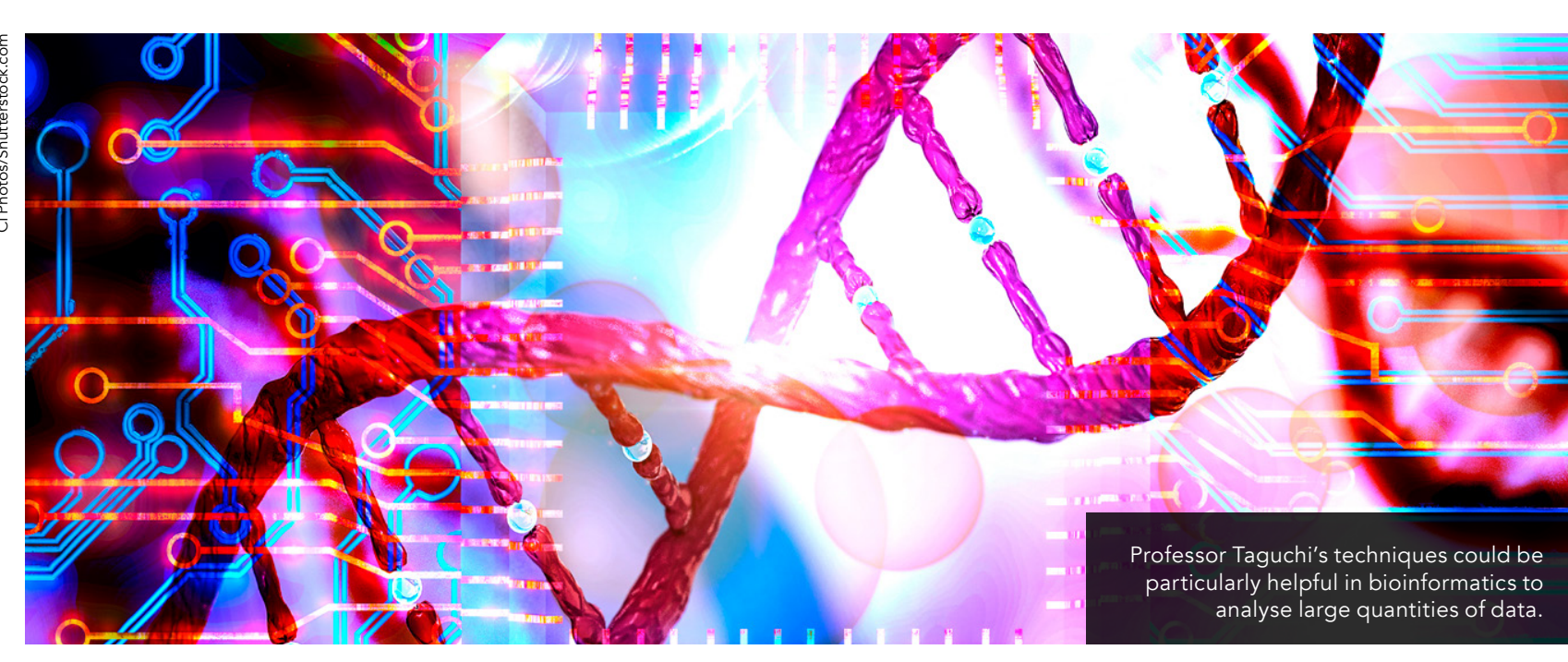

mathematical foundations through feature extraction techniques and on to the application of his concepts to varions in genomic science. they can be used demonstrates how they can be used to perform feature

Y-h Taguchi, from Chuo University, Tokyo, Japan, takes component analysis and tensor decomposition, and demonstrates how they can be used to perform feature selection in his cutting-edge research. Both unsupervised learning methods are applied to carry out feature extraction in a wide range of 'large $p$ small ${ }^{\prime}$ problems. This book dables the reader to analyse and many features. It offers a fast algorithm for analysing big data with output that is easily interpreted.

W $\begin{aligned} & \text { achine learning, an artificial } \\ & \text { intelligence (A) application that } \\ & \text { automates analytical model }\end{aligned}$ building for data analysis, has gained datasets. It is based on the concept that systems can learn, identify patterns and make decisions from data with little or no human intervention.

Machine learning techniques are powerful devices that benefit a wide spectrum of applications such as bioinformatics, where they are employed to explore the underlying mechanisms and interactions between biological molecules. They can also be used to predict the structure and function of proteins and identify disease-causing mutations. Moreover, they are essentia tools in the bi Supervised learning techniques, such as training dataset and have to achieve a satisfactory performance lovel before a new dataset is introduced. While popular, the application of supervised learning is limited as it requires an enormous amount of data. In contrast, unsupervised learning methods work on their own to discover patterns in the data and can perform more complex processing tasks.

\section{|IIll, I,Illi,Il,} Extraction Applied to Bioinformatics, Professor Y-h Taguchi, Professor of Physics at Chuo University, Tokyo, Jap
puts forward his novel applications
of unsupenvised learning Professor

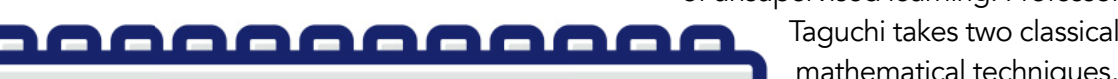

\section{EATURE SELECTION}

eature selection is the process of selecting a subset of relevant features, such as variables, attributes or predictors, from a dataset. Feature selection methods are trequently employed when there are a large number of features and relatively few samples to analyse. For example, it is often used in the analysis 位 or hundreds of samples contain many

\section{THE CURSE OF DIMENSIONALITY} Professor Taguchi is particularly interested in a sure selection proving the selection fra small number of critical variables ( problems. 'Large $\mathrm{p}^{\prime}$ refers to the massive 'promber of variables and 'smalln' massive the very small number of available observations. This predicament is known as 'the curse of dimensionality' which refers to the lack of enough observations when compared with the dimensionality i.e., the number of relevant features. This is a problem that has remal
unresolved for a long time.

\section{A JOURNEY FOR THE READER} Professor Taguchi has designed his book so that the intended audience, including students, researchers, and with ease. He has organ his concepts with ease. He has organised the book on a journey from the rudimentay

\section{MATHEMATICAL PREPARATIONS}

(n) The book starts with Professor Taguch algebra including scalars, vectors,

matrices, and from data science points of view. how vectors are

sets of scalars,

or real numerical ares, mposed (a) tensors are made up of matrices an goes on to demonstrate their arithme manipulation. The genertion of new scalars and vectors to represent descriptive features such as colours the cannot be defined with real values are covered too. These early chapters also contain exercises with worked solutions so the reader can practice the method and check their understanding.

The reader is then introduced to principal component analysis (PCA), as a part of matrix factorisation, and tensor decomposition (TD), two embedding techniques that can

\section{FEATURE EXTRACTION}

Professor Taguchi introduces feature extraction, a datadiven generator of the number of features in a dataset by creating new features from combinations of the original features, which are then features should summised set of

the original information. This differs from feature selection, which tends to rank the importance of the original features and

eatures can reduce the cost of individual observations. This means that the number can lead to a better outcome.

Even when cost is not an issue, feature selection is often preferabse, feature each feature maintains its own meaning. The author uses an example where and notes that it is more useful to have xtraction methods are powerful tools a limited number

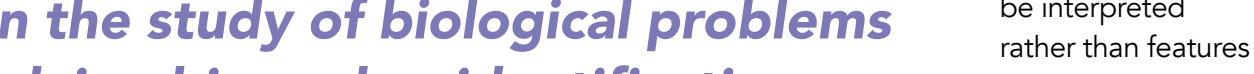
biomarker identification, gene combining a large xpression, and drug discovery.
APPLICATIONS TO the final section of his book introduction to genomic science before demonstrating the application of two methods, principal components analysis (PCA)-based unsupervised feature extraction and tensor decomposition (TD)-based unsupervised feature extraction, to large $\mathrm{p}$ small $\mathrm{n}$ problems in bioinformatics.

VS FEATURE SELECTION TS FEATURE SELECTION

extraction or feature selection is complex and depends highly on the context of the problem rather than mathematical cossor Taguchi's targeted field is bioinformatics and hexplains the reasons behind the bioinformatics andysis there is often a cost associated with measuring individual features, so measuring fewer
Bioinformatics focuses on the study of DNA, MRNA (messenger ibonucleic acid or RNA) and proteins. Much of the analysis in the latter chapters of this and miRNA expression data.

DNA methylation is a biological process nvolving the addition of methyl groups 


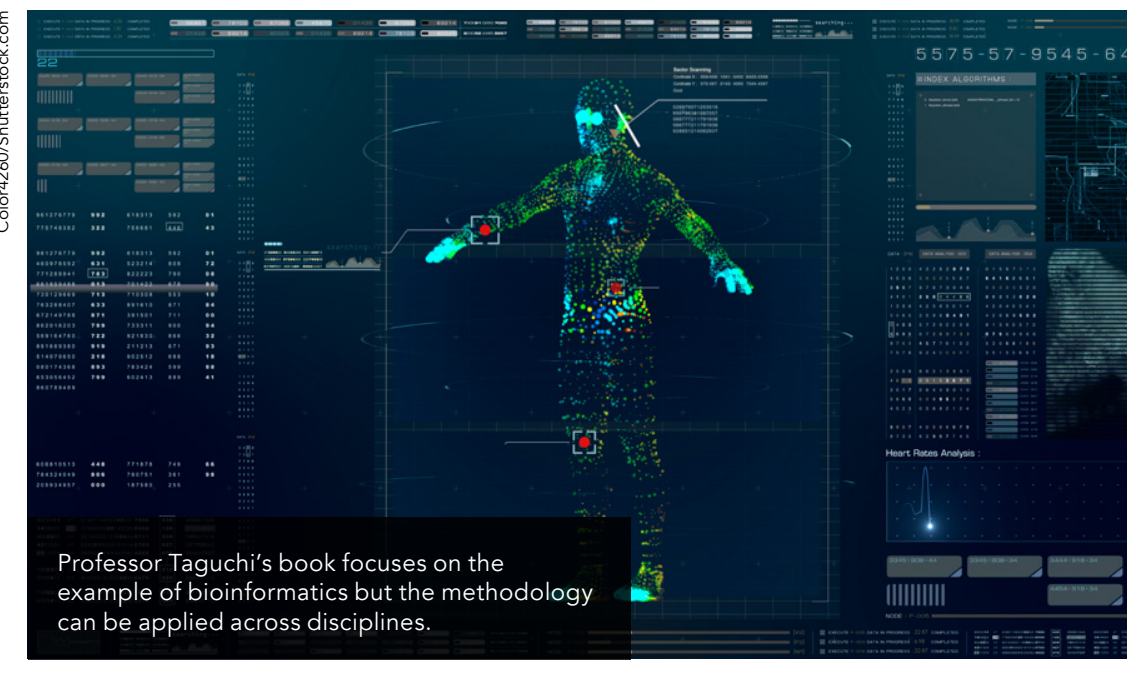

The methodology, based upon linear algebra, offers a fast algorithm for analysing big data with output that is easily interpreted.

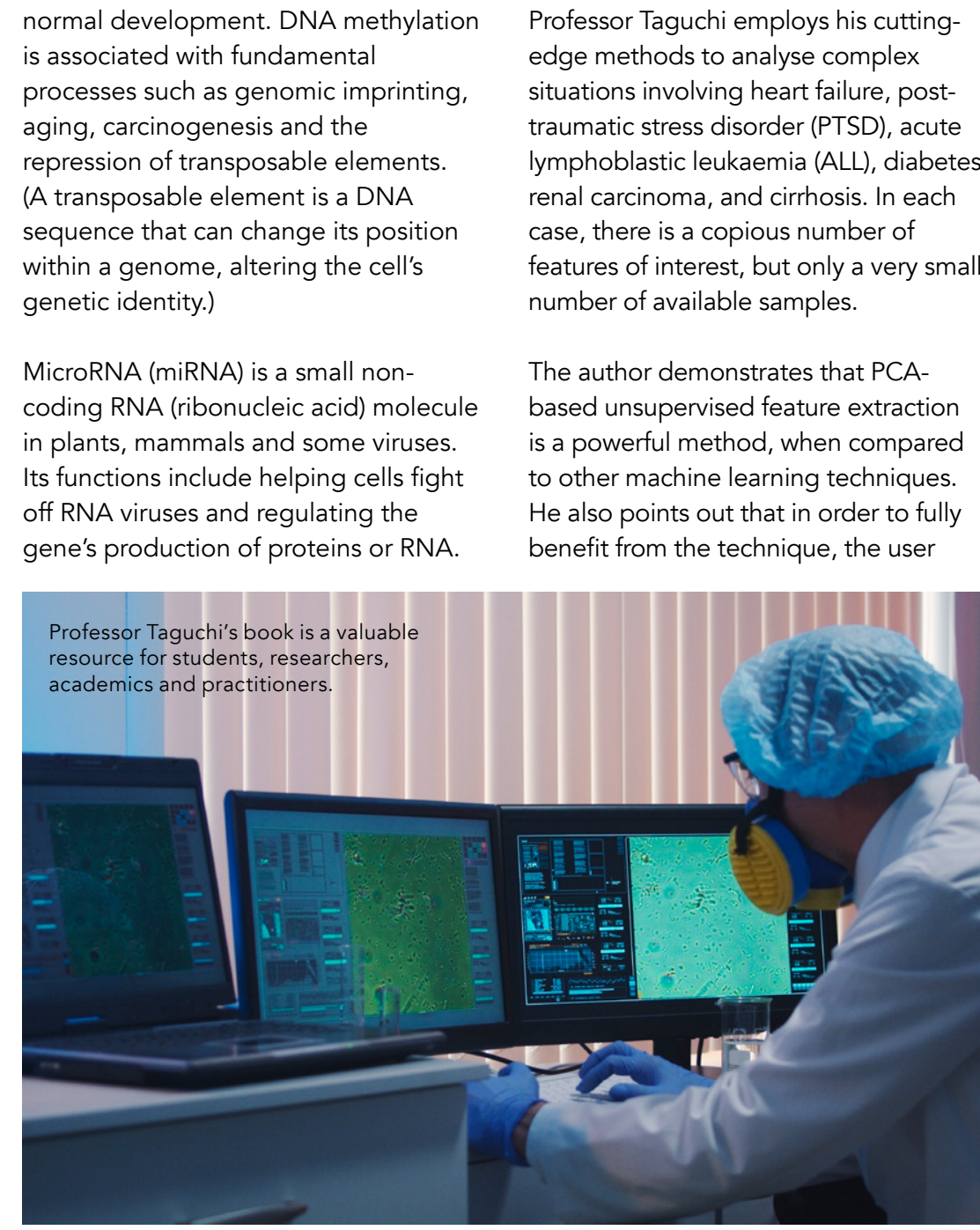

equires a deep understanding of the data to be analysed.

TD-based unsupervised feature extraction is a more recent development. Professor laguchi has applied it to many of the using PCA-based unsupervised feature extraction. He also mentions its new. application to in silico drug discovery.

ADVANTAGES OF UNSUPERVISED FEATURE EXTRACTION METHODS Professor Taguchi explains that when compared with conventional supervised methods, his unsupervised method are more robust. Both PCA-based unsupervised feature extraction and TDbased unsupervised feature extraction avoid the overfitting that can easily too closely to the training data and cannot $r$ to lise traing data and deal with unseen data. In addition, he points out that unsuperised methods are independent of labelling and therefore more stable. Furthermore, in both his PCA and TC methods, the interaction between variables is considered before, rather than after the features are selected.

\section{BROADER IMPACTS}

Professor Taguchi's methods can be applied to a wide range of large $p$

small $n$ problems enabling his readers to analyse data sets with small samples and many features. The data featros are easily interpreted as the output fertures The method features. The methodology, based upo analysing big data with output that is easily interpreted.

PCA and TD-based unsupervised feature extraction methods are powerful tools in the study of biological problems involving biomarker identification, gene expression, and drug discovery as demonstrated in this book and Professor Taguchi's numerous high-quality journa publications. This book is a valuable resource for students, researchers, academics and practitioners. Even though the applications demonstrated in the book focus on bioinformatics, the methodological framework is universal and can be ent

\section{Behind the Research}

Professor Y-h Taguchi

E: tag@granular.com T: +81-3-3817-1791 W: $\quad$ https://researchmap.jp/Yh_Taguchi/?lang=en

\section{Research Objectives}

Professor Taguchi proposes a new system of unsupervised learning for machine learning which would forticted

\section{Detail}

Y-h Taguchi

Partment of Physics

1-13-27 Kasuga

Bunkyo-ku

Tokyo 112-855

Japan

Bio

Professor Taguchi has been a physics professor at Chuo University, Tokyo, Japan since 2006. He received his Dr. Sci. from Tokyo Institute of Technology. He was an assistant professor there and moved to Chuo University in 1997. He was also a visiting professor at the European Bioinformatics Institute from 2009 to 2010.

Funding

Society for the Promotion of Science
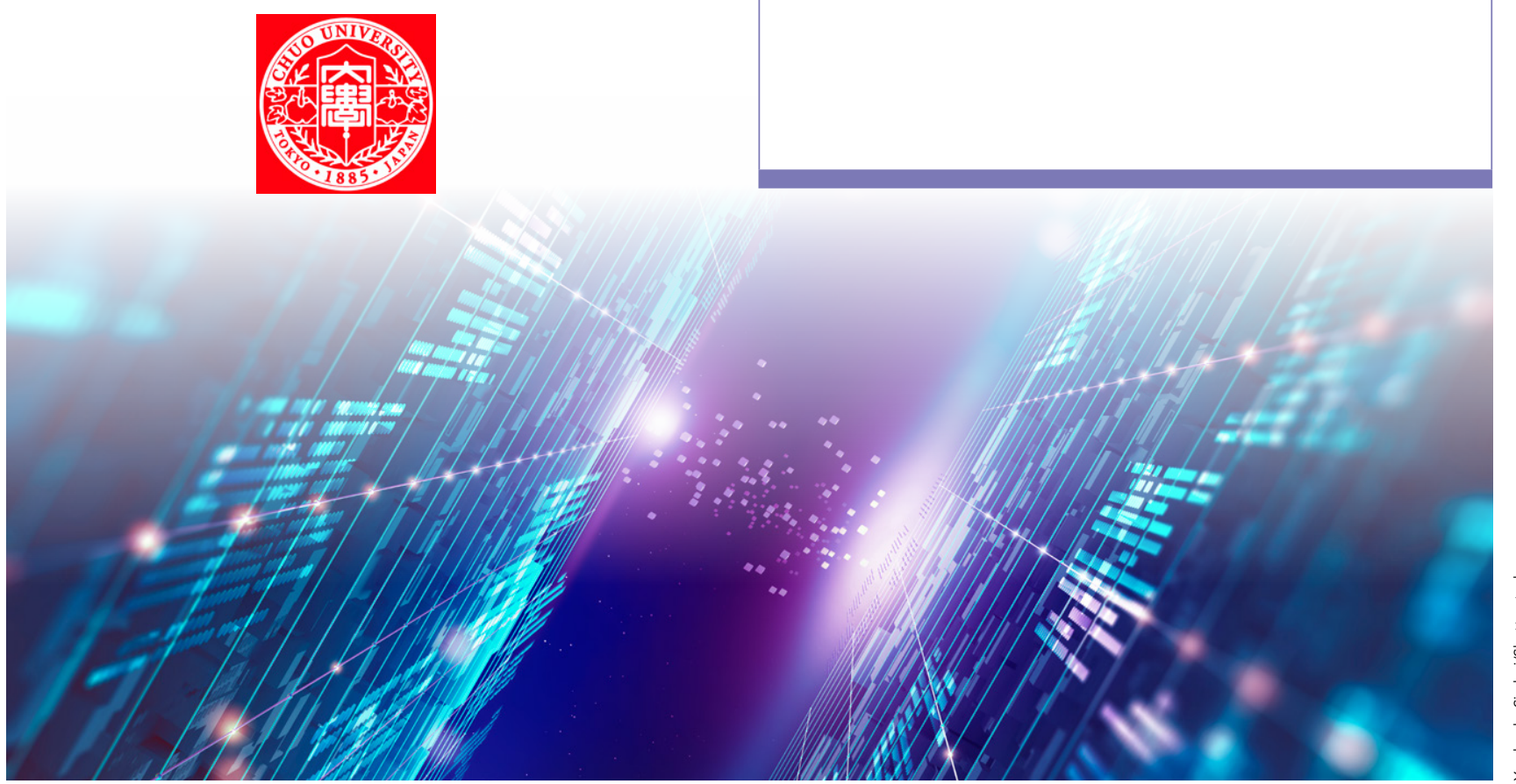

Taguchi, Y.H. (2020). Unsupervised Feature Extraction Approach, Springer, Switzerland. doi:10.1007/978-3-03022456-1. Available at: https://doi.org/10.1007/978-3-03022456-1 [Accessed 6th April 2020

\section{Personal Response}

What initially inspired your research into

II We human beings can have only limited intelligence since we are not God. Thus, any kind of supervised a data-driven approach can overcome our intelligence, since we do not make use of pre-knowledge.

One day, when I was testing numerous supervised surprised that it could tell me what I could never have predicted. I realised that if we can make further use of data-driven approaches, we can go beyond our own sense, an unsupervised approach is the closest method to God of date. Applied to Bioinformatics A PCA Based and TD Based 